This peer reviewed manuscript has been accepted for publications to the Tribology Letters. Cite this article as: Luis Bartolomé, Eva Oblak, Mitjan Kalin* Mechanical behaviour and constitutive models of ZDDP tribofilms on DLC coatings using nano-indentation data and finite element modelling.Tribology International 95 (2016) 19-26. DOI: https://doi.org/ 10.1016/j.triboint.2015.10.036

\title{
Mechanical behaviour and constitutive models of ZDDP tribofilms on DLC coatings using nano-indentation data and finite element modelling
}

\author{
Luis Bartolomé, Eva Oblak, Mitjan Kalin*
}

Laboratory for Tribology and Interface Nanotechnology, Faculty of Mechanical Engineering, University of Ljubljana, Bogišićeva 8, 1000 Ljubljana, Slovenia *Corresponding author: mitjan.kalin@tint.fs.uni-lj.si

Keywords: DLC, ZDDP, Finite-element method, AFM

The mechanical behaviour of zinc dialkyldithiophosphate (ZDDP) tribofilms on diamond-like carbon (DLC) coatings has been studied by combining the nano-indentation experimental data and finite-element modelling. Different constitutive models, whose analysis was performed using a global algorithm that consists of forward and inverse algorithms, were chosen to establish which of them more accurately predicts the actual mechanical behaviour of the ZDDP tribofilms. A non-homogeneous, dual structure, which was also verified by the local stiffness using the AFM force-modulation mode, was found. Based on the proposed algorithm, the mechanical properties of the ZDDP tribofilms were found, e.g. the elastic modulus $E=26 \mathrm{GPa}$ and the yield stress $Y=1.55 \mathrm{GPa}$ for the harder component and approximately $E=1.65 \mathrm{GPa}$ and $Y=0.144 \mathrm{GPa}$ for the softer component.

\section{Introduction}

Surface engineering technologies have experienced a significant progress during the last decade. The application of thin coatings, such as DLC, on the surfaces of engine components has provided significant improvements for the automotive industry, especially in terms of decreasing fuel consumption due to energy losses. DLC coatings lead to a reduction in friction, particularly in poor lubrication conditions. DLC coatings also provide an improvement in wear resistance due to their high hardness, high corrosion resistance and chemical stability [1]. Moreover, in boundary lubrication conditions, DLC coatings tend to form tribofilms, which also help in reducing both wear and friction [2].

Among the various automotive additives, ZDDP is one of the most indispensable and crucial due to its excellent anti-wear properties. The anti-wear performance of ZDDP in lubricated systems is related to the formation of a protective tribofilm [3]. ZDDP tribofilms have been studied widely for tribofilms formed on steel surfaces [2,3], mostly with respect to the tribological properties [4,5], but also with regards to the morphological [6,7], mechanical [8,9], kinetic [10,11] and thermal [12] properties. However, the tribofilms formed on DLC coatings have been studied less extensively. The studies of ZDDP tribofilms on DLC coatings are focused on the tribological [13-15] and morphological properties [16,17], but also on comparing ZDDP tribofilms on DLC coatings with ZDDP tribofilms on steel surfaces [18]. 
This peer reviewed manuscript has been accepted for publications to the Tribology Letters.

Cite this article as: Luis Bartolomé, Eva Oblak, Mitjan Kalin* Mechanical behaviour and constitutive models of ZDDP tribofilms on DLC coatings using nano-indentation data and finite element modelling.Tribology International 95 (2016) 19-26. DOI: https://doi.org/

10.1016/j.triboint.2015.10.036

The ZDDP tribofilms on DLC coatings have a different structure than the ZDDP tribofilms on steel surfaces due to the pad-like structures not being observed [16,18]. Moreover, the adhesion of ZDDP tribofilms is weaker on DLC coatings than on steel [14]. Some recent analyses of the mechanical properties and morphology revealed the complexity of ZDDP tribofilms, depending on the DLC coating and the lubricant's additives $[19,20]$. This complexity in structure has also been observed in some studies which are focused on molecular dynamics simulations of the ZDDP tribofilms [21]. However, the mechanical behaviour of ZDDP tribofilms on DLC coatings, especially a model that would make it possible to predict it, is not very well understood and remains absent from the literature, as well as a knowledge of how the mechanical properties affect the tribological behaviour of ZDDP tribofilms. Therefore, this work has focused on the mechanical behaviour of ZDDP tribofilms formed in boundary lubricated conditions on DLC coatings in order to establish a constitutive model to describe their mechanical behaviour.

Nano-indentation techniques have been widely used for studying the mechanical properties of engineering materials, coatings and films. The methods used to extract the mechanical properties from load-displacement, nano-indentation curves can be divided into two main categories [22].

The first category uses loading-unloading curves to extract the mechanical properties, extending the classic elastic solution of an infinite half space. In this category the method introduced by Oliver and Pharr [23] is extensively used for finding the elastic properties of materials, coatings and films [24-27]. Since this method does not take into account plasticity during the nano-indentation, a solution using a correction coefficient was proposed [28]. But even when using the correction coefficient, this method is unable for extracting the plastic properties.

The second category is the best for obtaining the various mechanical properties that the materials can exhibit. This second category uses intensive FE simulations for generating load-displacement curves for various combinations of mechanical properties and then trying to match the experimental data of the unknown material with the numerical results from the FE simulations to extract the mechanical properties of the analyzed material. The methods used in this category are mainly based on dimensional analysis for the inverse algorithms [29]. With these methods, different dimensionless parameters or functions are defined from the loading-unloading curve to solve the problem using iterative algorithms [30-32] or an incremental manner [33]. Most of the proposed methods use experimental data from the loading-unloading curves, but also the mechanical properties can be extracted using only loading curves [34-36]. Therefore, the analysis of the constitutive models for ZDDP tribofilms in this work is based on methods from this second category.

In this work, the loading curves of Berkovich indentations were used to stablish which mechanical behaviour, among linear elastic, elastic-plastic with or without hardening and nonlinear elastic, corresponds to the ZDDP tribofilms formed on DLC coatings. In order to extract the mechanical properties from the experimental nano-indentation data, an algorithm that consists of a forward algorithm and an inverse algorithm has been proposed. Using this 
This peer reviewed manuscript has been accepted for publications to the Tribology Letters.

Cite this article as: Luis Bartolomé, Eva Oblak, Mitjan Kalin* Mechanical behaviour and constitutive models of ZDDP tribofilms on DLC coatings using nano-indentation data and finite element modelling.Tribology International 95 (2016) 19-26. DOI: https://doi.org/

10.1016/j.triboint.2015.10.036

algorithm, the constitutive model that best fits the mechanical behaviour of the ZDDP tribofilms is obtained. We show that the ZDDP tribofilms on the DLC coatings are formed by two different material components, which have different mechanical behaviours. Therefore, each tribofilm component was studied separately. The softer component exhibits non-linear elastic with compressibility behaviour and is modelled using the neo-Hookean hyperelastic model. And the harder material exhibits elastic-plastic behaviour and is modelled using an elastic-plastic power law hardening model. Moreover, the mechanical properties of the ZDDP tribofilms on the DLC coatings were determined for both components. Therefore the ZDDP tribofilms on DLC coatings should be considered as a structured material with components that exhibit very different mechanical behaviours. This multi-structured behaviour should also be taken into account in the modelling of the ZDDP tribofilms.

\section{Methodology}

\subsection{Experimental methods}

In order to obtain the ZDDP tribofilms for the nano-indentation measurements, tribological tests were carried out using a tribometer (UMT-2, CETR, CA, USA). The test configuration was a ball on flat in reciprocating motion. The steel balls were rubbed against hydrogenated DLC (H-DLC) coated discs that were immersed in a solution of base oil (Group III, Lubrizol, OH, USA) suitable for automotive applications, with $1 \mathrm{wt} \%$ of ZDDP (Lubrizol, $\mathrm{OH}$, USA) providing the ZDDP tribofilms on DLC coatings. The H-DLC coating is a conventionally hydrogenated amorphous DLC coating with an sp3/(sp3+sp2) ratio from 35\% to $40 \%$ and a hydrogen content of 30 at. $\%$. The thickness of the H-DLC coating was $0.8 \mu \mathrm{m}$, with an additional two interlayers; Ti interlayer with $0.35 \mu \mathrm{m}$ and a Si-based interlayer with $0.6 \mu \mathrm{m}$ thick. The elastic modulus of the H-DLC coating is $151 \pm 3 \mathrm{GPa}$ and the hardness is $18.7 \pm 0.5 \mathrm{GPa}$. The oil has a viscosity of $76.6 \mathrm{~mm}^{2} / \mathrm{s}$ at $40{ }^{\circ} \mathrm{C}$ and $12.3 \mathrm{~mm}^{2} / \mathrm{s}$ at $100{ }^{\circ} \mathrm{C}$. The tests were performed at an elevated temperature of $100{ }^{\circ} \mathrm{C}$ to develop the ZDDP tribofilm on the DLC coating. The normal load was set to $10 \mathrm{~N}$, which corresponds to $1 \mathrm{GPa}$ of maximum initial Hertzian contact pressure. A frequency of $10 \mathrm{~Hz}$ and a stroke length of $5 \mathrm{~mm}$ were set, resulting in an average contact velocity of $0.1 \mathrm{~m} / \mathrm{s}$. The duration of the tests consisted of 15,000 cycles. After the tribological tests the discs were gently rinsed with a solvent (nheptane) to remove the excess oil, while retaining the adsorbed tribofilms on the surface.

In order to analyse, and determine the mechanical properties of the ZDDP tribofilms on the DLC coatings, nano-indentations were carried out with a Berkovich tip using a Nanoindenter (G200, Keysight Technologies, CA, USA). Using the Continuous Stiffness Measurement (CSM) mode, the loadings of the load-displacement curves were recorded. The nano-indentation measurements were performed on the surface of the tribofilm at several locations inside the wear track so as to achieve the most representative results. In order to avoid any influence of the substrate, the indentation depth should be set to approximately $10 \%$ of the thickness of the thin films [37]. Therefore, the indentation depth for was set to $20 \mathrm{~nm}$, due to the thickness of the ZDDP tribofilm was 175-200 nm, measured on the same films using an AFM (AFM CP-II, Brucker, CA, USA) [19]. 
This peer reviewed manuscript has been accepted for publications to the Tribology Letters.

Cite this article as: Luis Bartolomé, Eva Oblak, Mitjan Kalin* Mechanical behaviour and constitutive models of ZDDP tribofilms on DLC coatings using nano-indentation data and finite element modelling.Tribology International 95 (2016) 19-26. DOI: https://doi.org/

10.1016/j.triboint.2015.10.036

To verify and understand the nano-indentation data in more detail, the AFM force modulation mode (FMM), which provides local contrast for the mechanical stiffness properties of the surface, was used to evaluate the local mechanical behaviour on the surface of the ZDDP tribofilms. The FMM mode is based on the contact AFM mode; the feedback loop maintains a constant cantilever deflection, i.e. a constant force on the sample. The variation in the cantilever's deflection amplitude at the frequency of modulation is a measure of the relative stiffness of the surface. Therefore, the FMM imaging makes it possible to study the local mechanical properties of the surface, i.e. the relative differences in surface elasticity at nano-scale resolution [38]. The scan size of the FMM images, where the stiffness difference is shown by the brightness on the surface of the ZDDP tribofilms, was $5 \times 5 \mu \mathrm{m}^{2}$.

\subsection{Numerical methods}

In order to numerically determine the mechanical properties of the ZDDP tribofilms, an algorithm was defined (Fig. 1). This algorithm consists of a forward algorithm and an inverse algorithm, which should be performed sequentially.
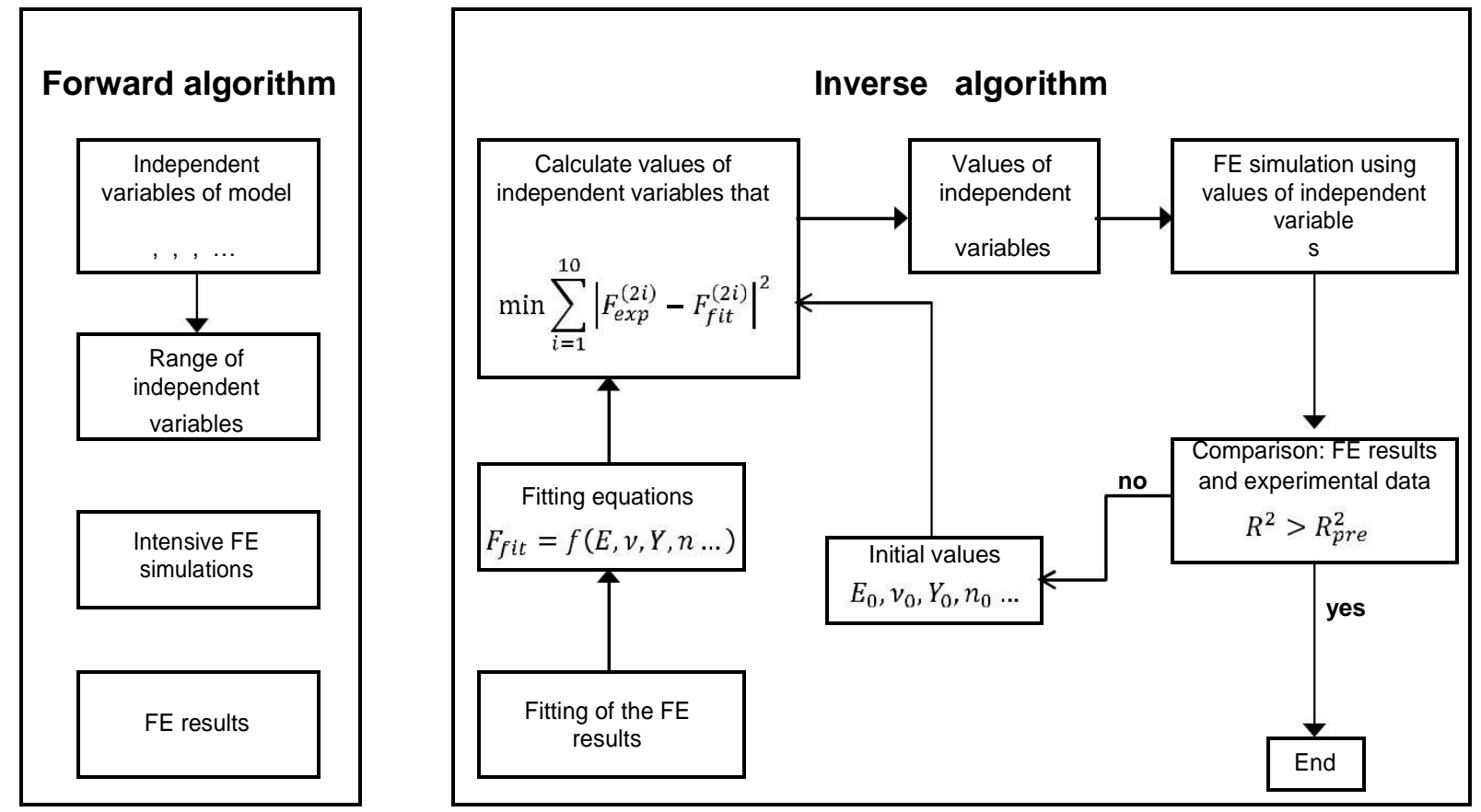

Figure 1: Algorithm for determining the mechanical properties of the ZDDP tribofilms.

The forward algorithm begins by establishing the independent variables or material properties of the constitutive model under consideration, e.g. if the linear elastic model is chosen to predict the mechanical behaviour of the material under investigation, then the independent variables are the elastic modulus and the Poisson's ratio. When the independent variables are established, a range of values is set for each independent variable, with enough intermediate values to provide a good fitting. But when the number of material properties is large, e.g. for elastic-plastic model obeying a power law, some of the material properties should be pre-set in order to ease the fitting process. This pre-setting can be accomplished by using constant values for some of the material's properties, the Poisson's ratio is usually fixed in order to determine the elastic-plastic properties of the coatings [31,32]. Otherwise, the presetting can be achieved by using alternative methods for extracting the mechanical properties 
This peer reviewed manuscript has been accepted for publications to the Tribology Letters.

Cite this article as: Luis Bartolomé, Eva Oblak, Mitjan Kalin* Mechanical behaviour and constitutive models of ZDDP tribofilms on DLC coatings using nano-indentation data and finite element modelling.Tribology International 95 (2016) 19-26. DOI: https://doi.org/

10.1016/j.triboint.2015.10.036

from the experimental data. Namely, Kuo and Huang [36] proposed that the yield stress can be identified using a double logarithm plot of the nano-indentation data that was used in this work. Following with the forward algorithm, intensive FE simulations were performed using all the possible combinations of the different values for the independent variables. During the numerical calculus, ten depths, from $2 \mathrm{~nm}$ to $20 \mathrm{~nm}$ in steps of $2 \mathrm{~nm}$, were defined along the loading curve. This solution of using intermediate points to determine the mechanical properties of thin films using only the loading curves was proposed by Zhao et al. [34]. The numerical results of the loads at the chosen indentation depths were saved in order to be used in the fitting process during the inverse algorithm (Fig. 2).

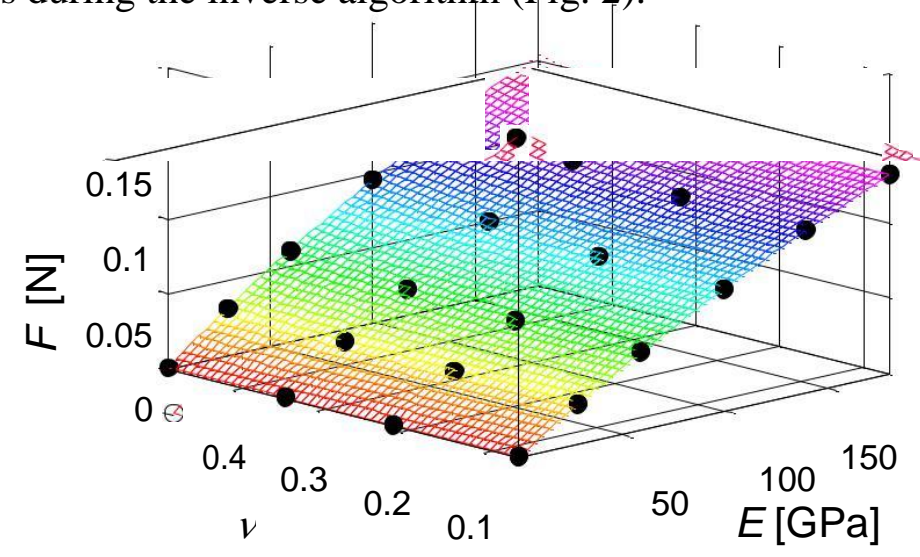

Figure 2: FE results of the maximum loads for the different combinations of $E$ and $v$ and the fitting surface using Eq. 1.

The inverse algorithm begins by fitting the FE results using polynomial equations for each specific point of the loading curve; therefore, ten equations are determined. Using these equations, the values of the independent variables that minimize the difference between the experimental data and the values from the fitting equations are calculated for all the specific points at the same time. These calculated values for the independent variables are implemented into the FE nano-indentation simulation again and the results of the loaddisplacement curve are compared with the experimental data. This process is continued using all the values that minimize the difference due to these values are highly dependent on the selected initial values and so multiple local minima may occur. The values of the independent variables that show the best correlation with the experimental data are chosen as a solution because the solution of the nano-indentation at moderate depths is unique according to Chen et al. [39]. Thereby the iterative algorithm finishes.

The numerical simulations of the nano-indentations for the algorithms were performed by means of the finite element method and the FE analysis was implemented using the commercial package Abaqus (version 6.13-4, ABAQUS Inc.). The nano-indentations were simulated using an axisymmetric model and these simulations were displacement-controlled. The ZDDP tribofilms were modelled as a flat body and the Berkovich indenter was simulated as a cone with a semi-angle of $70.3^{\circ}$, which gives the same area to depth relationship as a pyramidal Berkovich indenter [37]. In all the simulations, 4-node, bilinear, axisymmetric, quadrilateral, hybrid, constant pressure $(\mathrm{CAX} 4 \mathrm{H})$ elements were used to model the ZDDP tribofilm and the node bilinear axisymmetric quadrilateral (CAX4) elements were used to 
This peer reviewed manuscript has been accepted for publications to the Tribology Letters.

Cite this article as: Luis Bartolomé, Eva Oblak, Mitjan Kalin* Mechanical behaviour and constitutive models of ZDDP tribofilms on DLC coatings using nano-indentation data and finite element modelling.Tribology International 95 (2016) 19-26. DOI: https://doi.org/

10.1016/j.triboint.2015.10.036

model the Berkovich indenter. The mechanical properties of the ZDDP tribofilm were changed depending on the selected constitutive model, and the indenter $(E=1100 \mathrm{GPa}, v=0.07)$ was considered as an isotropic, linear elastic material. The model consists of 37120 elements for the ZDDP tribofilm and 20160 elements for the indenter. The normal displacement at the bottom of the ZDDP model was fixed. The reaction forces in the normal direction were recorded at every $2 \mathrm{~nm}$ of indentation depth between $2 \mathrm{~nm}$ and $20 \mathrm{~nm}$ for a comparison with the experimental data.

\section{Results}

\subsection{Experimental results}

The results of the experimental nano-indentations on the ZDDP tribofilms at the DLC coatings show two different and distinctive behaviours. These two behaviours are very consistently grouped in a clear mechanical response, as the experimental load-displacement curves show in Fig. 3. But due to the experimental measurements, both mechanical responses exhibit some scatter for both components of the ZDDP tribofilms on the DLC coatings. Therefore, an average for the experimental load-displacement curves was calculated for both components (see the dotted lines in Fig. 3). Clearly, one of the components of the ZDDP tribofilm is harder (high stiffness) than the other. These two average curves, one for the harder component and another for the softer component, were used to compare with the numerical results of the FE simulations.

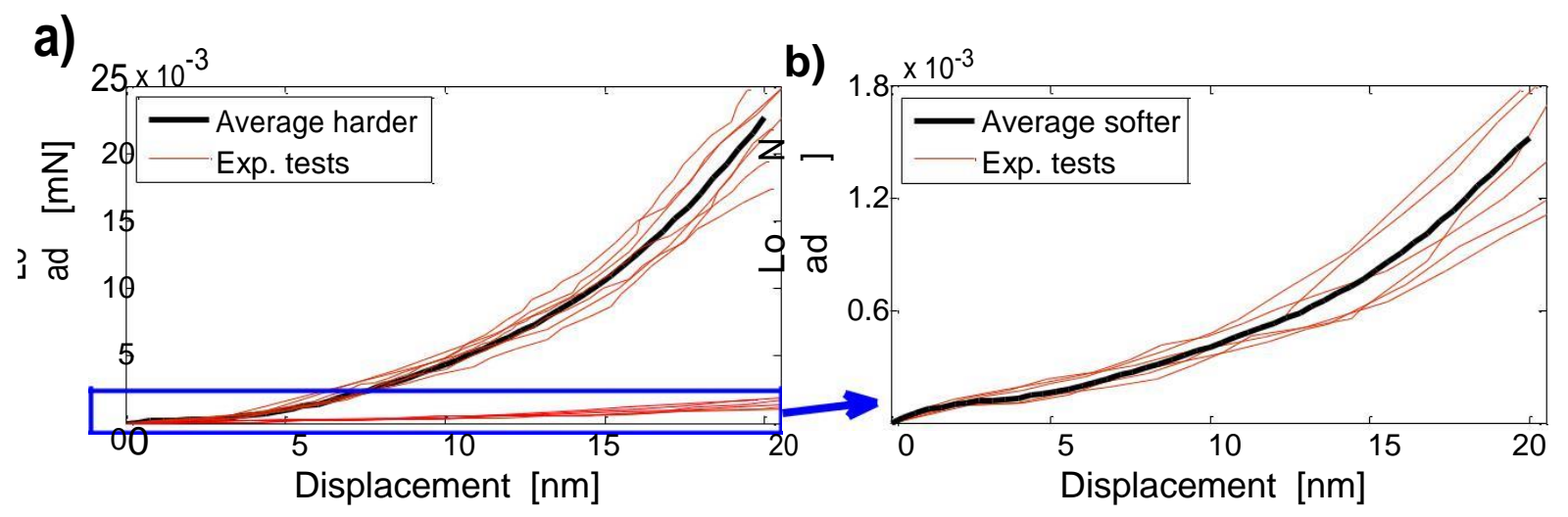

Figure 3: (a) Experimental displacement-load curves of the nano-indentations on the ZDDP tribofilms showing two distinctive mechanical responses and (b)augmentation for the softer component of the dual structure.

\subsection{Computational results}

\subsubsection{Linear elastic model}

When an isotropic linear elastic model is considered, two independent variables are required, i.e. the elastic modulus $E$ and the Poisson's ratio $v$. The values for the elastic modulus for the intensive simulations were 1, 30, 60100,140 and $180 \mathrm{GPa}$ and for the 
This peer reviewed manuscript has been accepted for publications to the Tribology Letters. Cite this article as: Luis Bartolomé, Eva Oblak, Mitjan Kalin* Mechanical behaviour and constitutive models of ZDDP tribofilms on DLC coatings using nano-indentation data and finite element modelling.Tribology International 95 (2016) 19-26. DOI: https://doi.org/

10.1016/j.triboint.2015.10.036

Poisson's ratio they were $0.07,0.22,0.35$ and 0.49 . The FE results for the normal load $F$ at different indentation depths $d$ were fitted using the following equation:

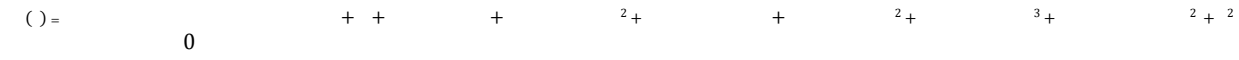

Using the proposed algorithm, the values for the best fitting of the elastic properties (elastic modulus and Poisson's ratio) are collected in Tables 1 and 2 for the harder and softer components, respectively. The errors for the mechanical properties were calculated by partially differentiating the fitting equations with respect to the independent variables and considering the largest error from among all the results obtained at the different loads. The correlations between the experimental data and the numerical solutions using the obtained values for the mechanical properties are shown in Fig 4.

Table 1: Mechanical properties for the harder component of the ZDDP tribofilms on the DLC coatings.

\begin{tabular}{|c|c|c|c|c|c|c|}
\hline Models & $\begin{array}{c}\text { Elastic } \\
\text { mod. } \\
E[\mathrm{GPa}]\end{array}$ & $\begin{array}{c}\text { Poisson's } \\
\text { ratio } \\
v \\
\end{array}$ & $\begin{array}{c}\text { Yield } \\
\text { stress } \\
Y[\mathrm{GPa}] \\
\end{array}$ & $\begin{array}{c}\text { Hardening } \\
\text { exp. } \\
n \\
\end{array}$ & $\begin{array}{c}\text { Material } \\
\text { param. } \\
C_{10} \\
\end{array}$ & $\begin{array}{c}\text { Material } \\
\text { param. } \\
D_{1} \\
\end{array}$ \\
\hline Linear elastic & $22.2 \pm 0.9$ & $0.34 \pm 0.02$ & & & & \\
\hline Elastic-perfectly plastic & $31 \pm 2$ & $0.31 \pm 0.02$ & $1.48 \pm 0.09$ & & & \\
\hline Elastic-plastic obeying power law & $26 \pm 1$ & $0.41 \pm 0.03$ & 1.55 & $0.52 \pm 0.05$ & & \\
\hline Neo-Hookean hyperelastic & & & & & $8.9 \pm 0.9$ & $15 \pm 2$ \\
\hline
\end{tabular}

Table 2: Mechanical properties for the softer component of the ZDDP tribofilms on the DLC coatings.

\begin{tabular}{|c|c|c|c|c|c|c|}
\hline Models & $\begin{array}{c}\text { Elastic } \\
\text { mod. } \\
E[\mathrm{GPa}]\end{array}$ & $\begin{array}{c}\text { Poisson's } \\
\text { ratio } \\
\text { v }\end{array}$ & $\begin{array}{c}\text { Yield } \\
\text { stress } \\
Y[\mathrm{GPa}] \\
\end{array}$ & $\begin{array}{c}\text { Hardening } \\
\text { exp. } \\
n \\
\end{array}$ & $\begin{array}{c}\text { Material } \\
\text { param. } \\
C_{10}\end{array}$ & $\begin{array}{c}\text { Material } \\
\text { param. } \\
D_{1} \\
\end{array}$ \\
\hline Linear elastic & $1.4 \pm 0.1$ & $0.41 \pm 0.02$ & & & & \\
\hline Elastic-perfectly plastic & $1.7 \pm 0.1$ & $0.39 \pm 0.02$ & $0.15 \pm 0.01$ & & & \\
\hline Elastic-plastic obeying power law & $1.65 \pm 0.09$ & $0.42 \pm 0.02$ & 0.144 & $0.21 \pm 0.02$ & & \\
\hline Neo-Hookean hyperelastic & & & & & $0.40 \pm 0.04$ & $5.0 \pm 0.6$ \\
\hline
\end{tabular}
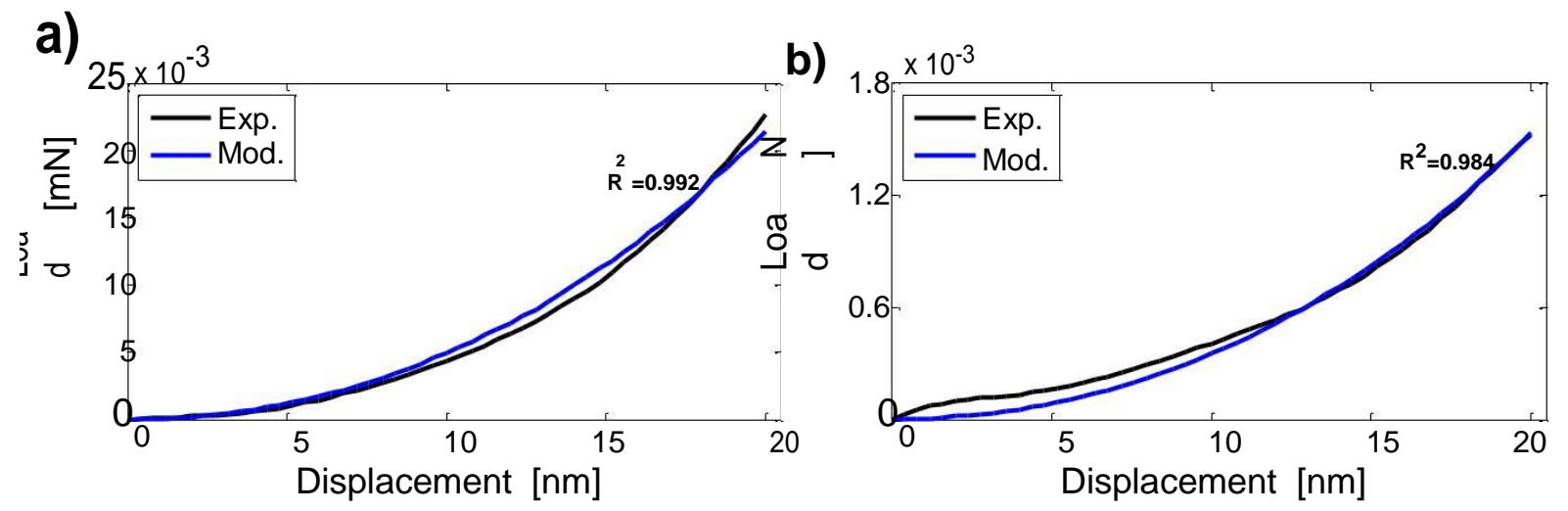

Figure 4: Comparison between the experimental nano-indentation data and the numerical displacementload results of the best fittings using the elastic model for the (a)harder component and the (b)softer component of the ZDDP tribofilm. 
This peer reviewed manuscript has been accepted for publications to the Tribology Letters.

Cite this article as: Luis Bartolomé, Eva Oblak, Mitjan Kalin* Mechanical behaviour and constitutive models of ZDDP tribofilms on DLC coatings using nano-indentation data and finite element modelling.Tribology International 95 (2016) 19-26. DOI: https://doi.org/

10.1016/j.triboint.2015.10.036

\subsubsection{Elastic-perfectly plastic model}

For the case of the isotropic elastic-perfectly plastic model, there are three independent variables to determine, the elastic modulus $E$ and the Poisson's ratio $v$ and the yield stress $Y$. In order to make the fitting process easier, different ranges for the independent variables were chosen, depending on the specific component of the ZDDP tribofilm. In the case of the harder component, the values of the ranges were: $10,25,40$ and $60 \mathrm{GPa}$ for the elastic modulus, 0.07 , $0.22,0.35$ and 0.49 for the Poisson's ratio and $0.5,1.5,3$ and $4.5 \mathrm{GPa}$ for the yield stress. For the softer component, the values of the ranges were: $0.3,1.25,2.5$ and $4 \mathrm{GPa}$ for the elastic modulus, $0.07,0.22,0.35$ and 0.49 for the Poisson's ratio and $0.01,0.25,0.5$ and $1 \mathrm{GPa}$ for the yield stress. The FE results for the normal load $F$ at different indentation depths $d$ for the harder component were fitted using the following equation:

And for the softer component the FE results were fitted using the equation:

$$
0^{++}
$$

For both components of the dual structure of the ZDDP tribofilm, the values of the best fitting using the proposed algorithm for the mechanical properties (elastic modulus, Poisson's ratio and yield stress) of the elastic-perfectly plastic model are collected in Tables 1 and 2. The correlations for the experimental data and the numerical solutions using these obtained values of the mechanical properties are shown in Fig 5.
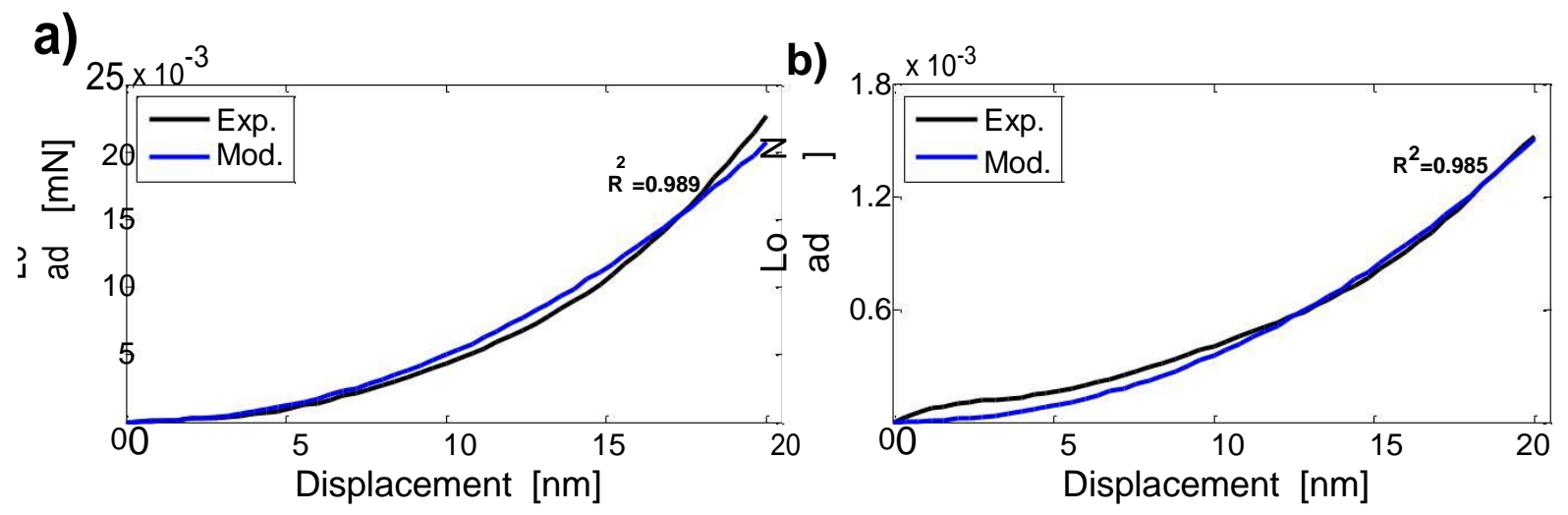

Figure 5: Comparison between the experimental nano-indentation data and the numerical displacementload results of the best fittings using the elastic-perfectly plastic model for the (a)harder component and the (b)softer component of the ZDDP tribofilm.

\subsubsection{Elastic-plastic model obeying a power law}

As there are four independent variables for the elastic-plastic model obeying a power law, i.e. the elastic modulus $E$ and the Poisson's ratio $v$, the yield stress $Y$ and the hardening exponent $n$, the fitting process could be complex. Therefore, the yield stress was pre-set to reduce the number of independent variables following the method proposed by Kuo and Huang [36], where the plot of the logarithm of the stress vs. double-logarithm of the displacement (Fig. 6) present two regions, one with a positive slope and another with a 
This peer reviewed manuscript has been accepted for publications to the Tribology Letters.

Cite this article as: Luis Bartolomé, Eva Oblak, Mitjan Kalin* Mechanical behaviour and constitutive models of ZDDP tribofilms on DLC coatings using nano-indentation data and finite element modelling.Tribology International 95 (2016) 19-26. DOI: https://doi.org/

10.1016/j.triboint.2015.10.036

negative slope. The minimum point in the plot corresponds to the depth at yielding (see Fig. 6). Therefore, the yield stress for the harder component is $1.55 \mathrm{GPa}$ and for the softer component it is $0.144 \mathrm{GPa}$.

After pre-setting the yield stress, only three independent variables need to be determined: the elastic modulus $E$, the Poisson's ratio $v$ and the hardening exponent $n$. As for elastic-perfectly plastic model, different ranges for the independent variables were chosen, depending on the specific component. In the case of the harder component, the values of the ranges were: 10, 25, 40 and $60 \mathrm{GPa}$ for the elastic modulus, $0.07,0.22,0.35$ and 0.49 for the Poisson's ratio and $0.1,0.25,0.4$ and $0.6 \mathrm{GPa}$ for the hardening exponent. For the softer component, the values of the ranges were: $0.3,1.25,2.5$ and $4 \mathrm{GPa}$ for the elastic modulus, $0.07,0.22,0.35$ and 0.49 for the Poisson's ratio and $0.1,0.25,0.4$ and $0.6 \mathrm{GPa}$ for the hardening exponent. The FE results for the normal load $F$ at different indentation depths $d$ for the harder component were fitted using the following equation:

and for the softer component the FE results were fitted using the equation:

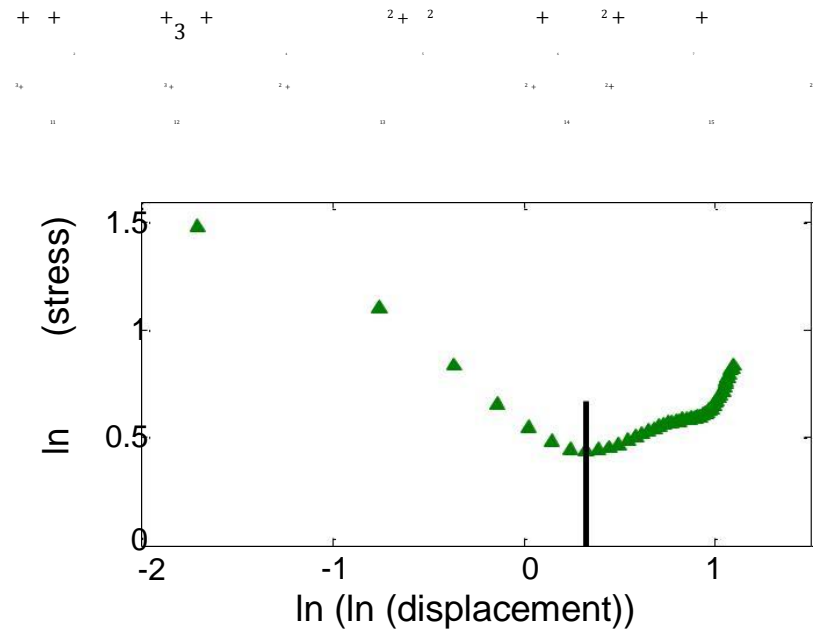

Figure 6: Logarithm of the stress vs. double-logarithm of displacement for the harder component.

The values of the best fitting using the proposed algorithm for the mechanical properties yet to be determined (elastic modulus, Poisson's ratio and hardening exponent) of the elastic-plastic model obeying a power law are collected in Tables 1 and 2 for both components, i.e. the harder and softer, respectively. The correlations of the experimental data and the numerical solutions using these obtained values of the mechanical properties are shown in Fig 7. 
This peer reviewed manuscript has been accepted for publications to the Tribology Letters. Cite this article as: Luis Bartolomé, Eva Oblak, Mitjan Kalin* Mechanical behaviour and constitutive models of ZDDP tribofilms on DLC coatings using nano-indentation data and finite element modelling.Tribology International 95 (2016) 19-26. DOI: https://doi.org/

10.1016/j.triboint.2015.10.036
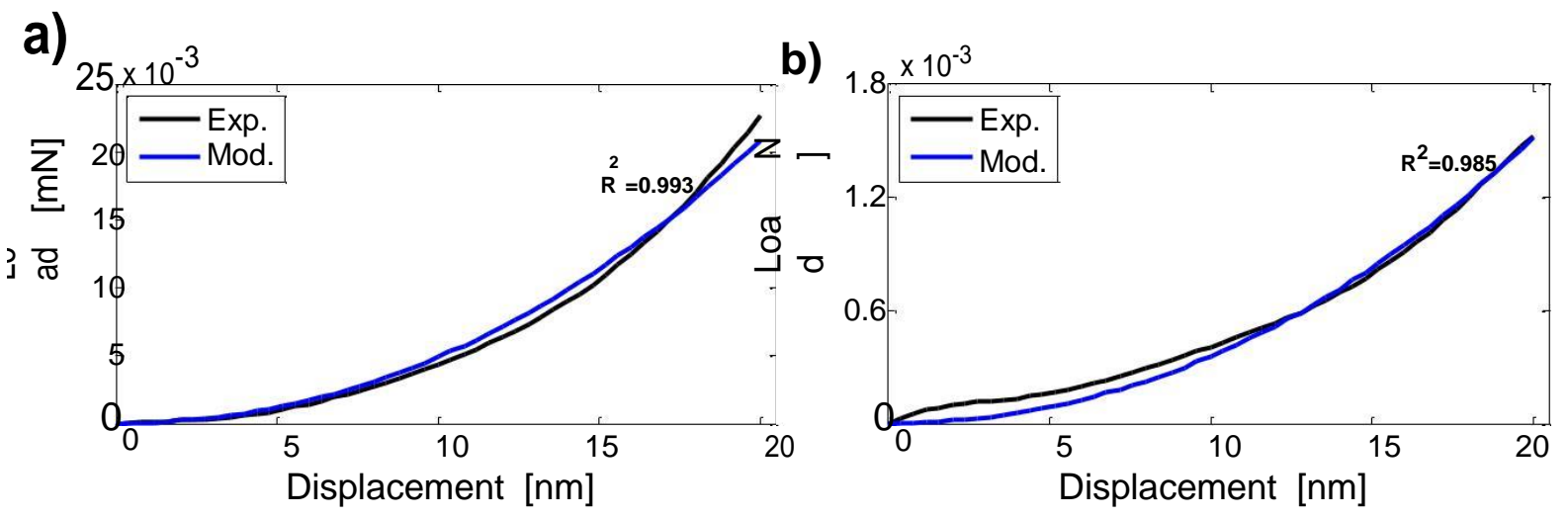

Figure 7: Comparison between the experimental nano-indentation data and the numerical displacementload results of the best fittings using the elastic plastic model obeying a power law for the (a)harder component and the (b)softer component of the ZDDP tribofilm.

\subsubsection{Non-linear elastic or hyperelastic model}

If the specific constitutive model is a neo-Hookean hyperelastic model, then two independent variables need to be determined, i.e. the material parameters $C_{10}$ and $D_{1}$, which are related to the initial shear modulus and the initial bulk modulus, respectively. Two different ranges of the independent variables were chosen depending on the specific component of the ZDDP tribofilm. In the case of the harder component, the values were 4, 8, 15 and $25 \mathrm{GPa}$ for $C_{10}$ and $5,15,25$ and $40 \mathrm{GPa}^{-1}$ for $D_{1}$. For the softer component, the values of $C_{10}$ for the intensive simulations were $0.1,0.6,1$ and $2 \mathrm{GPa}$ and of $D_{1}$ were $1,5,10$, 15 and $20 \mathrm{GPa}^{-1}$. The FE results for the normal load $F$ at different indentation depths $d$ for the harder component were fitted using the following equation:

And for the softer component the FE results were fitted using the equation:

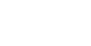

The values of the best fitting using the proposed algorithm for the material properties $C_{10}$ and $D_{1}$ of the neo-Hookean hyperelastic model are collected in Tables 1 and 2 for the harder and the softer components, respectively. The correlations of the experimental data and the numerical solutions using these obtained values for the mechanical properties are shown in Fig 8. 
This peer reviewed manuscript has been accepted for publications to the Tribology Letters. Cite this article as: Luis Bartolomé, Eva Oblak, Mitjan Kalin* Mechanical behaviour and constitutive models of ZDDP tribofilms on DLC coatings using nano-indentation data and finite element modelling.Tribology International 95 (2016) 19-26. DOI: https://doi.org/

10.1016/j.triboint.2015.10.036
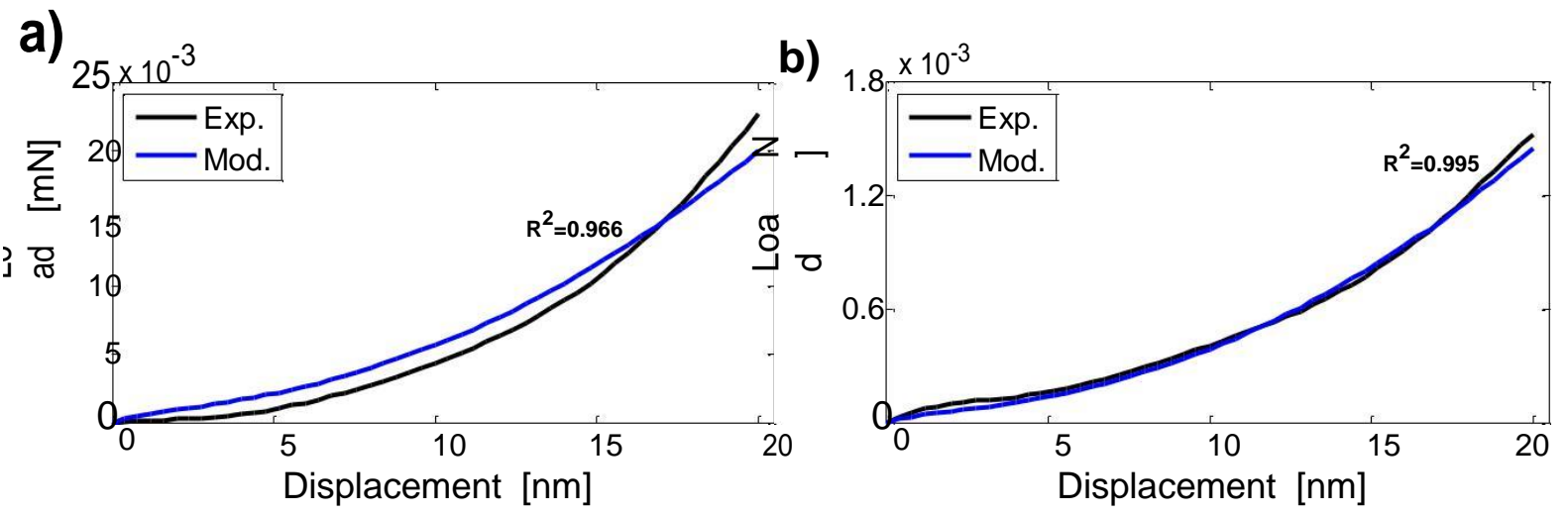

Figure 8: Comparison between the experimental nano-indentation data and the numerical displacement-load results of the best fittings using the neo-Hookean hyperelastic model for the (a)harder component and the (b)softer component of the ZDDP tribofilm.

\section{Discussion}

The experimental results of the nano-indentations on the ZDDP tribofilms clearly show two different mechanical responses (Fig 3), indicating that the ZDDP tribofilms on the DLC coatings consist of two distinctively different material components. This fact is in agreement with the literature about ZDDP tribofilms on steel, where a non-homogeneous structure is commonly observed [2-7]. In this work, the dual structure of the ZDDP tribofilm was additionally proved using AFM topography and the force modulation mode (FMM), which provides local contrast in the mechanical stiffness properties of the surface. Fig. 9a shows a topography image of a ZDDP tribofilm and Fig. 9b shows the corresponding image using FMM. In Fig. 9b the bright areas correspond to stiffer areas of the ZDDP tribofilm surface, while the dark areas represent the more elastic and more compliant components of the surface [38]. Focusing on the highlighted zones, i.e. the white contours, in Fig. 9, it is clear that these zones present dark and bright areas. This further confirms that the ZDDP tribofilm presents a dual structure with components that have two different stiffnesses. Furthermore, as Fig. 9b shows, both components of the dual structure are extended in a similar ratio over the surface, i.e. close to fifty-fifty for each tribofilm component.
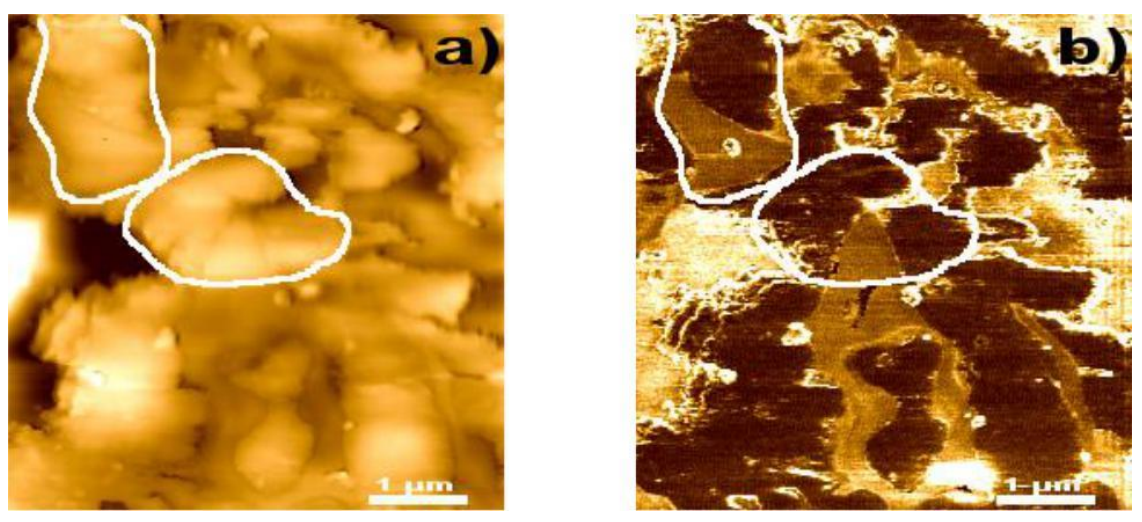
This peer reviewed manuscript has been accepted for publications to the Tribology Letters. Cite this article as: Luis Bartolomé, Eva Oblak, Mitjan Kalin* Mechanical behaviour and constitutive models of ZDDP tribofilms on DLC coatings using nano-indentation data and finite element modelling.Tribology International 95 (2016) 19-26. DOI: https://doi.org/

10.1016/j.triboint.2015.10.036

Figure 9: Images of ZDDP tribofilms on DLC coatings: (a)topography and (b)the corresponding stiffness map using FMM mode. 
This peer reviewed manuscript has been accepted for publications to the Tribology Letters.

Cite this article as: Luis Bartolomé, Eva Oblak, Mitjan Kalin* Mechanical behaviour and constitutive models of ZDDP tribofilms on DLC coatings using nano-indentation data and finite element modelling.Tribology International 95 (2016) 19-26. DOI: https://doi.org/

10.1016/j.triboint.2015.10.036

The chemical and, especially, the mechanical structure of ZDDP tribofilms on DLC coatings are still not well explored and are the subject of on-going investigations [19, 20, 40]. Some variations in the observed characteristics of the previous results could also be attributed to differences in the DLC coatings, oils, additives and experimental contact conditions that clearly affect the properties of the tribofilms [19]. Further detailed chemical analyses of these films on DLC are an on-going research activity for us [20]; however, earlier studies of ZDDP tribofilms on steel [3] and DLC [40], also found structured tribofilms and suggested that the harder component corresponds to layers of sulphites or phosphates, while precipitates from the degradation process and wear could provide lubricious properties [3,8]. Therefore, it seems that these earlier results agree very well with the structure and properties of the ZDDP tribofilms found in current work, although the actual chemistry of the tribofilms is not yet completely revealed.

The findings in this work, however, confirm that the ZDDP tribofilms on DLC coatings cannot be described by a single, unique, mechanical response, but that ZDDP tribofilms present multiple components that exhibit different mechanical behaviours. In particular, this should be considered when the proportion of different components is significant, like in tribofilms in this study. Therefore, the ZDDP tribofilms should be considered as a structured material where the mechanical properties of the components could differ by as much as an order of magnitude (see Tables 1 and 2). For example, if the elastic modulus and the yield stress are considered, the harder component has an elastic modulus $E=26 \mathrm{GPa}$ and a yield stress $Y=1.55 \mathrm{GPa}$, while the softer component exhibits $E=1.65 \mathrm{GPa}$ and $Y=0.144 \mathrm{GPa}$. The mechanical behaviour of these multi-structured ZDDP tribofilms on DLC coatings is relatively complex, depending on type of DLC coatings, additives and sliding distances, which have been described in morphological and chemical terms in more detail elsewhere $[19,20]$. Therefore, the mechanical modelling of the ZDDP tribofilms on DLC coatings becomes a complex task because they do not exhibit homogenous mechanical properties.

With regards to the constitutive models for the components of the ZDDP tribofilms on DLC coatings, the linear elastic model would not be appropriate for predicting the mechanical behaviour of the tribofilm components because it does not take into account the plastic deformation that is always presented due to the high local stresses on the asperities during the contact. Moreover, plastic deformation is also present in the nano-indentations with Berkovich tip, which were measured and simulated in this work using the proposed algorithm (Fig.1). In this way when the plasticity is considered, the FE results using an elastic-plastic model obeying a power law present the best correlation with the experimental data for the harder component (see Fig. 7a). In the case of the softer component, none of the FE results using linear elastic models with plasticity provided a good correlation with the experimental data (see Fig. 5b and Fig. 7b). Therefore, the neo-Hookean model, i.e. the isotropic non-linear elastic or hyperelastic model, was used in this work, which, however, showed a very good correlation with the experimental data (see Fig. 8b). Therefore the mechanical behaviour of the softer component exhibits non-linear elastic behaviour with compressibility. 
This peer reviewed manuscript has been accepted for publications to the Tribology Letters. Cite this article as: Luis Bartolomé, Eva Oblak, Mitjan Kalin* Mechanical behaviour and constitutive models of ZDDP tribofilms on DLC coatings using nano-indentation data and finite element modelling.Tribology International 95 (2016) 19-26. DOI: https://doi.org/

10.1016/j.triboint.2015.10.036

\section{Conclusions}

The conclusions drawn from this study are as follows:

- The ZDDP tribofilms on DLC coatings present a dual structure with two material components that exhibit two significantly different mechanical behaviours. This was revealed by the nano-indentation tests, as well as by the AFM topography and the local stiffness properties.

- The harder component of the ZDDP tribofilms exhibits linear elastic-plastic behaviour and the softer component exhibits non-linear elastic behaviour with compressibility.

- The mechanical behaviour of the ZDDP tribofilms is predicted accurately using the elastic-plastic model obeying a power law for the harder component and using the neo-Hookean hyperelastic model for the softer component.

- The mechanical properties of the studied ZDDP tribofilms on DLC coatings were as follow: elastic modulus $E=26 \mathrm{GPa}$ and yield stress $Y=1.55 \mathrm{GPa}$ for the harder component and approximately $E=1.65 \mathrm{GPa}$ and $Y=0.144 \mathrm{GPa}$ for the softer component

- The ZDDP tribofilms on DLC coatings cannot be considered as a homogeneous material with a unique mechanical behaviour, but as a structured material with components that exhibit very different mechanical behaviours. This multi-structured behaviour should also be taken into account in the modelling of the ZDDP tribofilms.

\section{Acknowledgements}

This study was funded by the FP7 program through the Marie Curie Initial Training Network (MC-ITN) entitled "ENTICE - Engineering Tribochemistry and Interfaces with a Focus on the Internal Combustion Engine" [290077] and was carried out at University of Ljubljana. The authors would like to thank to all ENTICE partners for kind discussions on the topic and the methodology.

\section{References}

[1] Kalin M, Velkavrh I, Vižintin J, Ožbolt L. Review of boundary lubrication mechanisms of DLC coatings used in mechanical applications. Meccanica 2008;43:623-37.

[2] Spikes H. The history and mechanisms of ZDDP. Tribol Lett 2004;17:469-89.

[3] Bec S, Tonck A, Georges JM, Coy RC, Bell JC, Roper GW. Relationship between mechanical properties and structures of zinc dithiophosphate anti-wear films. Proc Royal Soc Lond A: Math, Phys Eng Sci 1999;455:4181-203.

[4] McQueen JS, Gao H, Black ED, Gangopadhyay AK, Jensen RK. Friction and wear of tribofilms formed by zinc dialkyl dithiophosphate antiwear additive in low viscosity engine oils. Tribol Int 2005;38:289-97. 
This peer reviewed manuscript has been accepted for publications to the Tribology Letters. Cite this article as: Luis Bartolomé, Eva Oblak, Mitjan Kalin* Mechanical behaviour and constitutive models of ZDDP tribofilms on DLC coatings using nano-indentation data and finite element modelling.Tribology International 95 (2016) 19-26. DOI: https://doi.org/

10.1016/j.triboint.2015.10.036

[5] Wang Y, Yue W, She D, Fu Z, Huang H, Liu J. Effects of surface nanocrystallization on tribological properties of 316L stainless steel under MoDTC/ZDDP lubrications. Tribol Int 2014;79:42-51.

[6] Aoki S, Masuko M, Suzuki A. Application of ellipsometry to the characterization of reaction films formed by ZnDTPs on sliding steel surfaces. Tribol Int 2007;40:1454-65.

[7] Topolovec-Miklozic K, Forbus TR, Spikes HA. Film thickness and roughness of ZDDP antiwear films. Tribol Lett 2007;26:161-71.

[8] Ye J, Kano M, Yasuda Y. Evaluation of local mechanical properties in depth in modtc/zddp and zddp tribochemical reacted films using nanoindentation. Tribol Lett 2002;13:41-7.

[9] Nicholls M, Bancroft GM, Norton P, Kasrai M, De Stasio G, Frazer B et al. Chemomechanical properties of antiwear films using X-ray absorption microscopy and nanoindentation techniques. Tribology Letters 2004;17:245-59.

[10] Fujita H, Glovnea RP, Spikes HA. Study of zinc dialkydithiophosphate antiwear film formation and removal processes, Part I: Experimental. Tribol Trans 2005;48:558-66.

[11] Morina A, Neville A. Understanding the composition and low friction tribofilm formation/removal in boundary lubrication. Tribol Int 2007;40:1696-704.

[12] Demmou K, Bec S, Loubet J, Martin J. Temperature effects on mechanical properties of zinc dithiophosphate tribofilms. Tribol Int 2006;39:1558-63.

[13] Kalin M, Roman E, Vižintin J. The effect of temperature on the tribological mechanisms and reactivity of hydrogenated, amorphous diamond-like carbon coatings under oil-lubricated conditions. Thin Solid Films 2007;515:3644-52.

[14] Equey S, Roos S, Mueller U, Hauert R, Spencer ND, Crockett R. Tribofilm formation from ZnDTP on diamond-like carbon. Wear 2008;264:316-21.

[15] Abdullah Tasdemir H, Wakayama M, Tokoroyama T, Kousaka H, Umehara N, Mabuchi $\mathrm{Y}$ et al. The effect of oil temperature and additive concentration on the wear of nonhydrogenated DLC coating. Tribol Int 2014;77:65-71.

[16] Vengudusamy B, Green JH, Lamb GD, Spikes HA. Tribological properties of tribofilms formed from ZDDP in DLC/DLC and DLC/steel contacts. Tribol Int 2011;44:165-74.

[17] Mabuchi Y, Higuchi T, Inagaki Y, Kousaka H, Umehara N. Wear analysis of hydrogenfree diamond-like carbon coatings under a lubricated condition. Wear 2013;298-299:48-56.

[18] Equey S, Roos S, Mueller U, Hauert R, Spencer ND, Crockett R. Reactions of zinc-free anti-wear additives in DLC/DLC and steel/steel contacts. Tribol Int 2008;41:1090-6. 
This peer reviewed manuscript has been accepted for publications to the Tribology Letters. Cite this article as: Luis Bartolomé, Eva Oblak, Mitjan Kalin* Mechanical behaviour and constitutive models of ZDDP tribofilms on DLC coatings using nano-indentation data and finite element modelling.Tribology International 95 (2016) 19-26. DOI: https://doi.org/

10.1016/j.triboint.2015.10.036

[19] Oblak E, Kalin M. Relationship between the nano-scale topographical and mechanical properties of tribochemical films on DLC coatings and their macroscopic friction behavior. Submitted to Tribol Lett (2015).

[20] Kalin M, Oblak E, Akbari, S. Evolution of tribofilm nano-scale mechanical properties during sliding formed on DLC coatings. Unpublished results.

[21] Onodera T, Kuriaki T, Morita Y, Suzuki A, Koyama M, Tsuboi H et al. Influence of nanometer scale film structure of ZDDP tribofilm on Its mechanical properties: A computational chemistry study. Appl Surf Sci 2009;256:976-9.

[22] Heinrich C, Waas AM, Wineman AS. Determination of material properties using nanoindentation and multiple indenter tips. Int J Solids Struct 2009;46:364-76.

[23] Oliver WC, Pharr GM. An improved technique for determining hardness and elastic modulus using load and displacement sensing indentation experiments. J Mater Res 1992;7:1564-83.

[24] Chen S, Liu L, Wang T. Investigation of the mechanical properties of thin films by nanoindentation, considering the effects of thickness and different coating-substrate combinations. Surf Coat Technol 2005;191:25-32.

[25] Guruvenket S, Li D, Klemberg-Sapieha JE, Martinu L, Szpunar J. Mechanical and tribological properties of duplex treated TiN, nc-TiN/a-SiNx and nc-TiCN/a-SiCN coatings deposited on 410 low alloy stainless steel. Surf Coat Technol 2009;203:2905-11.

[26] Zyganitidis I, Kalfagiannis N, Logothetidis S. Ultra sharp Berkovich indenter used for nanoindentation studies of TiB2 thin films. Mater Sci Eng: B 2009;165:198-201.

[27] Wheeler JM, Curran JA, Shrestha S. Microstructure and multi-scale mechanical behavior of hard anodized and plasma electrolytic oxidation (PEO) coatings on aluminum alloy 5052. Surf Coat Technol 2012;207:480-8.

[28] Oliver WC, Pharr GM. An improved technique for determining hardness and elastic modulus using load and displacement sensing indentation experiments. J Mater Res $1992 ; 7: 1564-83$.

[29] Cheng Y, Cheng C. Scaling, dimensional analysis, and indentation measurements. Mater Sci Eng: R: Reports 2004;44:91-149.

[30] Zhao M, Chen X, Yan J, Karlsson AM. Determination of uniaxial residual stress and mechanical properties by instrumented indentation. Acta Materialia 2006;54:2823-32.

[31] Chen X, Yan J, Karlsson AM. On the determination of residual stress and mechanical properties by indentation. Mater Sci Eng: A 2006;416:139-49.

[32] Yan J, Chen X, Karlsson AM. Determining equi-biaxial residual stress and mechanical properties from the force-displacement curves of conical microindentation. J Eng Mater Technol 2006;129:200-6. 
This peer reviewed manuscript has been accepted for publications to the Tribology Letters. Cite this article as: Luis Bartolomé, Eva Oblak, Mitjan Kalin* Mechanical behaviour and constitutive models of ZDDP tribofilms on DLC coatings using nano-indentation data and finite element modelling.Tribology International 95 (2016) 19-26. DOI: https://doi.org/

10.1016/j.triboint.2015.10.036

[33] Dao M, Chollacoop N, Van Vliet KJ, Venkatesh TA, Suresh S. Computational modeling of the forward and reverse problems in instrumented sharp indentation. Acta Materialia 2001;49:3899-918.

[34] Zhao M, Xiang Y, Xu J, Ogasawara N, Chiba N, Chen X. Determining mechanical properties of thin films from the loading curve of nanoindentation testing. Thin Solid Films 2008;516:7571-80.

[35] Zheng YP, Choi APC, Ling HY, Huang YP. Simultaneous estimation of Poisson's ratio and Young's modulus using a single indentation: a finite element study. Meas Sci Technol 2009;20:045706.

[36] Kuo J, Huang I. Extraction of plastic properties of aluminum single crystal using Berkovich indentation. Mater Trans 2010;51:2104-8.

[37] Fischer-Cripps AC, Nanoindentantion. New York: Springer Science+Business Media LLC; 2011.

[38] Li F, Thompson GE, Newman RC. Force modulation atomic force microscopy: background, development and application to electrodeposited cerium oxide films. Appl Surf Sci 1998;126:21-33.

[39] Chen X, Ogasawara N, Zhao M, Chiba N. On the uniqueness of measuring elastoplastic properties from indentation: The indistinguishable mystical materials. J Mech Phys Solids 2007;55:1618-60.

[40] Vengudusamy B, Green J, Lamb G, Spikes H. Durability of ZDDP Tribofilms Formed in DLC/DLC Contacts. Tribol Lett 2013;51:469-78. 